\section{VHe 04}

INFLUENCE OF RECOMBINANT HUMAN INTERFERON-GAMMA ON PLATELET FUNCTION AND COAGULATION FACTORS IN VITRO AND IN PATIENTS TREATED FOR HEMATOLOC IG MAL IGNANCIES D. Böllert, H.H. Bartsch, H. Köstering, K. Pfizenmaier and G.A. Nage 1

A large number of cell functions in normal and malignant cells are influenced by interferons such as induction of MHC-genes, specific intracellular enzymes as $2^{\prime} 5^{\prime}$ oligoadenylatcyclase or suppression of cellular oncogenes. Since more and more phase II trials with IFN's not only as monotherapy, but also in combination with cytotoxic agents are designed, it is of prefrential interest to investigate a possible influence of interferon on platelet function and coagulation factors. In a recent study (J.Mirro et al., Cancer Treat. Rep. 69:315, 1985) it was demonstrated for the first time that IFN-alpha-treatment did induce a coagulopathy in children with myelocytic leukemia. Prolonged activated partial thromboplastin time and depressed activity of factor II, VII, IX, X and XII were demonstrated. Since we started a phase II trial with recombinant human Interferon-gamma in patients with hematologic malignancies, we were interested in the question, whether IFN-gamma might influence platelet function or coagulation factors. Therefore, we investigated platelet function by ADP Collagen- and Ristocetin-induced platelet aggregation as we11 as platelet factor 3 . Platelet rich plasma from normal donors were incubated for $30 \mathrm{~min}$ with different doses of IFN-gamma, $\mathrm{NaCl}$ or salicylic acid. There was no significant difference in platelet aggregation in the IFN-gamma group as compared to $\mathrm{NaCl}$, while salicylic acid caused dramatically reduced aggregation, as expected. Additionally, we measured the activity of several coagulation factors (II, VII, IX, X, XII, XIII) under the same conditions without evidence to be influenced by IFN-gamma.

Div. Hematology/Oncology, Dept. of Medicine, University of Göttingen, Robert-Koch-Str. 40, 3400 Göttingen, F.R.G.

\section{VHe 05}

PLATELET SECRETION AND THROMBIN GENERATION IN MALIGNANCY R.E. Scharf, 0 . Theis and W. Schneider

To assess whether or not a relationship between hemostatic disorders and extent or activity of malignant proliferation exists, we studied 38 pts. $(49+15$ yrs.) with histologically proven neoplasia (carcinoma $n=24$; malignant melanoma $n=5$; malignant lymphoma $n=9$ ), but without bleeding. Platelet activity and thrombin generation were evaluated by plasma levels of $B$-thromboglobul in (BTG), p1atelet factor 4 (PF4) and fibrinopeptide A (FPA). - The mean level of FPA $(7.1+$ 5.1 , range $1.7-17.5 \mathrm{ng} / \mathrm{ml}$ ) was significantly increased $i \bar{n}$ tumor pts. (normal range $0-2.5 \mathrm{ng} / \mathrm{ml}, \mathrm{p}<0.001$ ). Fourteen pts. with malignancy revealed pathologically increased levels of $B T G(>30 \mathrm{ng} / \mathrm{ml})$ and PF4 $(>6 \mathrm{ng} / \mathrm{ml})$; their mean concentration of BTG was $49+11 \mathrm{ng} / \mathrm{m}$, that of PF4 $12+6 \mathrm{ng} / \mathrm{ml}$. These data differed significantly from those of $3 \overline{8}$ normals (BTG $19+7, \mathrm{PF} 44+2 \mathrm{ng} / \mathrm{ml}$ ), or those of the other $24 \mathrm{pts}$. wi th maTignancy $T B T G 19+8, P F 44+2 \mathrm{ng} / \mathrm{ml}, \mathrm{p}<0.001$ ), respectively. However, when dividing the total group of pts. in (A) subjects with progressive disease $(n=20)$ and in $(B)$ those with limited disease or those with remission $(n=18)$, there was no significant difference between mean plasma levels $(\mathrm{ng} / \mathrm{ml})$ of BTG $(A: 31+17, B: 24+16)$, PFA (A: $7+5, B$ : $8+6)$ or FPA $(A: 7.0+4.9, B: 8.3+5.7)$. The sensitivity and specifity of $B T G$ and PF4 measurements were tested in subgroup A (i.e. pts. with extensive disease). In 9 of $20 \mathrm{pts}$. with tumor progression a significant increase of BTG and PF4 occured revealing a sensitivity of $45 \%$. The specifity or the prognostic value of significantly elevated BTG and PF4 plasma levels (i.e. percentage of rea17y abnormal data related to tumor progression) was $64 \%$. - This study confir ms that activation of platelets and coagulation occurs in neoplastic disease. However, the sensitivity and specifity of platelet secretion and thrombin generation are rather low related to the extent of tumor progression. We therefore conclude that measurements of BTG, PF4 and FPA do not allow any estimation of the disease activity in individual patients with solid tumors or malignant lymphomas.

Med. Univ.-Klinik, Moorenstr. 5, D 4000 Duisseldorf, F.R.G.
VHe 06

LEU 7 (HNK 1) -A MARKER FOR SURFACE-DERIVED EPITHELIAL CELLS IN THYMOMA AND THYMIC CARCINOMA Th. Kirchner and H.K. Müller-Hermelink

Thymic epithelial tumours can be divided in thymomas and thymic carcinomas. Based on light microscopical and immunohistochemical features of the normal thymus, thymomas of cortical, medullary and mixed types were distinguished. Other authors previously observed, that the Leu-7-antigen is expressed in normal thymic surface epithelium and also in some epithelial cells of thymomas. For this reason we investigated the reactivity of 6 epithelial thymic tumours comprising 5 thymomas and 1 thymic carcinoma with anti-Leu-7 (Becton-Dickinson, USA) using the PAP-method on frozen sections.

No expression of Leu-7-antigen was found in 2 thymomas of common mixed type, but all 3 thymomas of cortical or predominant cortical type included Leu-7-positive cells. In 1 of these cases anti-Leu-7 was reactive only at the lobule boundary. 2 thymomas of predominant cortical type showed a reticular and focal confluent staining of epithelial cells with a conspicious lining of positive cells around perivascular spaces. The most extented Leu-7-reactivity was observed in the epithelial cells of a well differentiated thymic carcinoma, in which Leu-7 + cells formed sheets bordering perivascular spaces. These findings suggest, that the Leu $7+$ thymic surface epithelium may represent the proliferating cell layer for some types of thymomas or thymic carcinomas and that the intensity of the Leu-7-expression may indicate the amount of less differentiated surface epithelium derived tumor cells.

Pathologisches Institut der Universität,JosefSchneider-Str. 2, D-8700 würzburg

\section{VHe 07}

Ploidy pattern of megakaryocytes in patients with metastatic tumors and in controls M.Winkelmann, W.Schneider, P.Pfitzer

An altered platelet heterogeneity could be one of the reasons for thrombotic events in malignancy. Although there is evidence that this heterogeneity depends on the different ploidy of megakaryooytes very little is known about these cells in patients with malignant tumors.

We studied the DNA-content of megakaryocytes of the following patients: 30 patients with metastatic tumors - 15 with and 15 without paraneoplastic thrombosis, 15 patients with localized cancer, 15 patients with thromboses but without tumors, and 75 healthy controls.

In both groups of patients with metastatic tumors a largely increased ploidisation of the megakaryocytes with an average ploidy index of 3.54 and 3.56 compared with 3.06 of the controls was found. Ploidy index of patients with localized cancer was 3.18 and the ploidy index of patients with thromboses only was 3.15. This means that patients with metastatic tumors have significantly (p<0.0001) increased ploidy of megakaryocytes independent of whether they suffer from thromboses or not. This shift is less pronounced in patients with localized cancer implying a possible correlation between ploidisation of the megakaryocytes and tumor mass. Thrombosis alone does not alter ploidy patterns significantly (p<0.1).

The consequence of higher ploidisation of megakaryocytes is a larger cytoplasmic mass of these cells. This means an increased platelet production and leads to an alteration in the heterogeneity of circulating platelets. A thus altered platelet population could favour but not necessarily provoke thrombotic complications in malignancy. Besides an overcompensation for increased platelet consumption a mitogenic or thrombopoietin-like factor produced by the tumor itself or by macrophages must be considered.

Abteilung Hämatologie, Onkologie und klin. Immunologie, Universität Düsseldorf, Moorenstr. 5, 4000 Düsseldorf 